\title{
Le lait et l'allaitement dans le discours égyptien sur la constitution du corps
}

Youri VOLOKHINE

Département des sciences de l'Antiquité, Unité d'histoire et d'anthropologie des religions,

Faculté des Lettres, Université de Genève,

2 rue de Candolle, $\mathrm{CH}-1211$ Genève 3 (Suisse)

youri.volokhine@unige.ch

Publié le 30 juin 2017

MOTS CLÉS

Égypte pharaonique, allaitement,

Pharaon allaité, vaches divines, physiologie égyptienne, anthropologie pharaonique, constitution du corps en

Égypte ancienne.

KEY WORDS

Pharaonic Egypt, suckling,

Pharao's suckling,

divine cows,
milk,

Egyptian physiology, pharaonic anthropology, body's constitution in
Ancient Egypt.
Volokhine Y. 2017. - Le lait et l'allaitement dans le discours égyptien sur la constitution du corps, in Arena F., FoehrJanssens Y., Papaikonomou I. \& Prescendi F. (éds), Allaitement entre humains et animaux: représentations et pratiques de l'Antiquité à aujourd'hui. Anthropozoologica 52 (1): 83-90. https://doi.org/10.5252/az2017n1a7

\section{RÉSUMÉ}

L'étude de plusieurs motifs mettant en scène un «allaitement interspécifique», dont celui du pharaon allaité, permet de comprendre la relation particulière que nouent les déesses et le souverain qui s'alimente à leur sein ou à leur mamelle. L'examen des scènes figurées révèle une théologie de l'allaitement et du lait, autour de la transmission de la vie par un fluide divin. Les différents aspects revêtus par la déesse allaitante conduisent également à s'interroger sur la nature du corps dans les conceptions propres à l'Égypte pharaonique, et à réfléchir sur la part masculine et féminine dans sa constitution ainsi qu'au rôle du lait dans la physiologie pharaonique.

\section{ABSTRACT}

Milk and suckling in the Egyptian discourse on the constitution of the body.

The study of several motives about "interspecific feeding", among which that of the breast-fed Pharaoh, allows to understand the particular relation which tie some goddesses and the sovereign, who feeds in their breast (or udder). The examination of the figurative scenes reveals a theology of the feeding and of the milk, around the transmission of life by a divine fluid. The various aspects taken on by the breast-feeding goddess also lead to wonder about the nature of the body in the conceptions of Pharaonic Egypt, and to precise Egyptian ideas about the male and feminine parts in the constitution of the body, and also about the role of the milk in the pharaonic physiology. 


\section{INTRODUCTION}

Le motif de la vache divine allaitant le roi est fort bien connu dans l'iconographie égyptienne; l'image est frappante: le roi boit directement à la mamelle de l'entité divine thériomorphe. L'analyse de cette image offre l'avantage de poser clairement la question de la symbolique de l'allaitement divin, qui engage celle, plus générale encore, du lait. En prenant appui sur ce motif, l'exposé qui va suivre propose une vision synthétique du discours égyptien sur les fluides corporels et l'allaitement. Pour saisir ce que cet allaitement particulier signifie, il s'agit de retracer dans ses grandes lignes la théologie égyptienne du lait, et de comprendre le rôle de ce liquide dans la construction du corps. Ce thème conduit à envisager différents éléments d'un discours sur le corps, dont la question des frontières imaginées entre parties solides et parties molles, frontières marquées respectivement par la théorie égyptienne des genres masculin et féminin. Le lait et l'allaitement demandent à être compris dans le mécanisme général des fluides corporels imaginé par la physiologie symbolique égyptienne. On tentera donc ici de préciser le rôle que le lait joue dans ce discours.

Un tableau schématique concernant les motifs (iconographiques et narratifs) attestant de scènes qui montrent des animaux ou des divinités thériomorphes en situation d'allaiter des humains, et vice versa, permet de résumer succinctement la situation (Tableau 1).

Avant de revenir sur les différents cas, on peut d'emblée poser que:

- en contexte social, l'allaitement «interspécifique» est une rareté en Égypte ancienne. Les rares exemples sont connus par l'iconographie funéraire (paragraphe "Allaitement» entre humains et animaux en situation sociale). L'allaitement au sein d'un jeune animal par une femme est inconnu;

- le thème de l'allaitement du roi par une divinité est au contraire un motif symbolique courant (paragraphe Allaitement en situation symbolique);

- il existe plusieurs divinités qui, sous formes anthropomorphes ou thériomorphes, sont figurées en train d'allaiter un dieu ou le roi (paragraphe Le roi allaité par une entité thériomorphe).

Ces cas invitent nécessairement à traiter de la symbolique du lait, dont on esquissera quelques expressions mythologiques (paragraphe Le lait et les chairs), et dont on résumera les conséquences dans le cadre de la physiologie du corps (paragraphe La chair et les os, le lait et le sperme). Pour une vision complète de la question, on peut renvoyer à la récente monographie consacrée à la mère, à l'enfant et au lait dans la culture pharaonique, qui offre une utile synthèse et une vaste documentation (Jean \& Loyrette 2010).

\section{"ALLAITEMENT» ENTRE HUMAINS ET ANIMAUX EN SITUATION SOCIALE}

De rares scènes figurées dans des tombes du Moyen Empire pourraient attester du fait que l'on pouvait à l'occasion s'abreuver directement à la mamelle d'un animal. Ainsi, dans la tombe de Baqet à Beni Hasan (no 15), on surprend un paysan s'abreuvant au pis d'une vache (Griffith \& Newberry 1893: pl. VII; Shedid 1994: 29; Kanawati \& Woods 2010: 77, photo 164). La petite taille du personnage n'indique pas forcément qu'il soit un enfant (il n'en présente d'ailleurs pas les traits conventionnels). La vache tourne sa tête vers son veau, écarté de la mamelle. Une scène similaire est probablement aussi figurée dans une tombe d'une nécropole d'El Bersheh (Griffith \& Newberry 1894: pl. 14). À proprement parler, il ne s'agit pas d'allaitement, mais tout au plus d'une prise directe de lait au pis. Ces scènes expriment-elles une idée particulière liée à la consommation de lait, ou sont-elles des illustrations «ethnographiques» d'une pratique en contexte agricole, un détail naturaliste saisi sur le vif? Il faut peut-être d'abord évaluer la portée de la représentation. En premier lieu, ces illustrations tirées d'un répertoire funéraire tendent à mettre en valeur le propriétaire de la tombe à laquelle elles appartiennent. Ce qui est représenté doit renvoyer vraisemblablement à une situation liée au prestige social, qu'il s'agit de mettre en valeur. Pour l'essentiel, ce discours vise les membres de la communauté locale qui pouvaient visiter cette partie de la tombe. L'usage du lait est très largement documenté en Égypte ancienne. Le lait de vache est de loin le plus fréquent, mais on connaît aussi des laits de chèvre, de brebis et autres, lesquels servaient probablement surtout pour la confection des fromages (Lefebvre 1960); le lait, présenté en cruche, figure habituellement dans les scènes d'offrande. On a pu dire qu'il était une boisson de «tous les jours» (Guglielmi 1982). Il est bien attesté dans les recettes médicales, sous différentes formes (Bardinet 1995: 574, s.v. «lait»). Il faut néanmoins prendre en considération le fait que le lait non cuit - Sinouhé boit du lait cuit (Grandet 1998: 19) - ne se conserve pas bien sous sa forme liquide, et tourne rapidement, d'autant plus dans un climat brûlant comme celui de l'Égypte. On pourrait donc proposer d'interpréter la représentation du paysan buvant «à la mamelle» comme un signe de la jouissance sans entrave d'un lait toujours frais, qui "coule à flots». En ajoutant ce détail dans le programme iconographique de la tombe, on procède probablement à renforcer encore le prestige du défunt, par le biais de l'évocation de l'heureuse richesse de ses domaines agricoles.

Dans le sens inverse (humain - animal), le don éventuel de lait offert à une jeune bête sevrée n'est soutenu que par quelques rares témoignages. Toutefois, dans tous les cas, il ne s'agit pas strictement d'allaitement, mais de nourrissage. À l'Ancien Empire, une scène du mastaba de Kagemeni montre un petit mammifère qui semble ainsi nourri de bouche à bouche par un humain (Harpur \& Scremin 2006: 62). Plutôt que d'y voir un geste tendre (un baiser), l'on a habituellement considéré qu'il s'agissait d'une sorte d'allaitement. On a parfois vu l'animal comme un porcelet, mais ses traits caractéristiques semblent plutôt être ceux d'un petit chien: le museau pointu, les oreilles molles, la queue dressée, les quatre griffes bien distinctes, semblent plutôt caractériser un chiot-tchésem (Volokhine 2014: 83, 84). Il faut là aussi tenir compte de la nature idéologique du discours monumental porté par ces scènes funéraires. 
Ce qui est montré n'est pas forcément la représentation d'une scène familière, mais plutôt l'expression d'une idée visant à valoriser le propriétaire du monument. Ici, l'on voudrait peut-être signifier à quel point l'on s'est soucié de l'entretien des bêtes, en allant jusqu'à les nourrir au bouche à bouche. Notons également qu'une petite $(6 \mathrm{~cm})$ statuette en faïence de la XIIIe dynastie, au Musée Myers d'Eton (Boysson \& Spurr 2007: no 16, ECM 1841), montre un jeune chiot semblablement "allaité» par un nain, ce qui tend encore à signaler que l'on évolue bien dans le registre spécifique du discours sur la fécondité (la forme naine étant liée au monde symbolique de la prospérité). Dans ces deux cas, le nourrissage du jeune animal se pratique au bouche à bouche; on peut raisonnablement supposer que l'aliment offert est du lait. À ma connaissance, aucun cas d'allaitement au sein impliquant une femme et un jeune animal ne sont connus en situation sociale, à la différence de ce que l'ethnologie atteste ailleurs (Millet 1987).

\section{ALLAITEMENT EN SITUATION SYMBOLIQUE}

Déjà dans les Textes des Pyramides (Sethe 1908-1922), le plus ancien corpus funéraire égyptien, le statut du lait comme offrande divine est valorisé (paragraphes $89 \mathrm{~b}$ et $131 \mathrm{~d}$ ); le lait y est également personnifié par une déesse Iat, «Le Lait» (Leclant 1951). Le lait participe du principe égyptien de la pureté (ouâb) ; tant sa blancheur (couleur favorable) que sa nature nourricière contribuent à son grand prestige symbolique. De manière plus générale, le lait intervient dans le discours égyptien sur les fluides corporels, auquel il contribue de manière significative (Spieser 2014). Les mythes lui confèrent des vertus de guérison magique. Dès la XVIIIe dynastie, le principe personnifiant le lait devient d'ailleurs masculin, et c'est désormais un dieu "Lait», Iati, qui va se trouver figurer dans le contexte de "l'allaitement royal " portant sur sa tête une cruche à lait. Peu de documents permettent d'évaluer son rôle et tout porte à croire que cette entité spécialisée intervenait surtout dans les rituels royaux. Rien n'indique que le dieu "Lait» ait été l'objet de faveurs populaires. Par ailleurs, des dieux bouviers président aussi au don du lait, comme le dieu laitier et vacher Tjeni, ou encore certaines formes d'Anubis bouvier (Quaegebeur 1977). Le lait et les offrandes lactées sont en bonne place dans les scènes des temples d'époque gréco-romaine, où l'on évoque toute la gamme des douces valeurs lactées, blancheur, pureté et suavité (Erroux-Morfin 2009: 130-133). Par ailleurs, les scènes d'allaitement sont nombreuses dans l'iconographie consacrée à la femme et à l'enfance, qui comporte toujours de fortes connotations symboliques (Rigault 2013). La focalisation iconographique sur ce geste tendre, exprimant de manière sensible et humaine la protection maternelle, connaît un grand essor dans l'iconographie divine. Le développement au cours du premier millénaire avant notre ère des cultes osiriens, et simultanément celui des formes juvéniles divines, mettent en avant les "Horus enfants » : jeune dieu héritier, parfois figuré en triomphateur et souvent égale-
TABLEAU 1. - Motifs iconographiques et narratifs attestant de scènes qui montrent des animaux ou des divinités thériomorphes en situation d'allaiter des humains.

\begin{tabular}{lc}
\hline $\begin{array}{c}\text { Animal allaitant directement } \\
\text { (à la mamelle) un humain }\end{array}$ & $\begin{array}{c}\text { Humain allaitant directement } \\
\text { (au sein) un animal }\end{array}$ \\
\hline \multicolumn{2}{c}{ En situation sociale } \\
Motif très rare (homme buvant & Motif inconnu \\
au pis) & \\
\hline \multicolumn{2}{c}{ En situation symbolique } \\
Déesse thériomorphe allaitant & $\begin{array}{c}\text { Déesse anthropomorphe allaitant } \\
\text { un dieu ou le roi. Motif très }\end{array}$ \\
$\begin{array}{cc}\text { un dieu ou le roi. Motif très } & \text { courant. } \\
& \text { Déesse anthropomorphe allaitant } \\
& \text { un dieu zoomorphe. Motif bien } \\
& \text { attesté. }\end{array}$ \\
\hline
\end{tabular}

ment en bambin sur les genoux de sa mère Isis qui l'allaite. À l'époque gréco-romaine, cette Isis lactans est devenue l'une des formes internationales (diffusée dans le cadre des cultes isiaques méditerranéens) d'un binôme Isis/Horus célébrant tant la protection de la vie que la transmission du pouvoir (Tran Tam Tinh 1973). Un cas particulier de l'iconographie de l'allaitement divin est aussi offert par des représentations frappantes de la déesse Neith anthropomorphe, allaitant deux crocodiles accrochés respectivement à ses seins (par exemple sur le "torse de Naples", El Sayed 1982: 469, 470). Les deux crocodiles sont probablement les rejetons de cette Neith démiurgique, soit Chou et Tefnout, soit plus vraisemblablement encore Senouy (divinité dont le nom sous forme de duel apparent peut se comprendre comme "Les deux frères "), père du saurien Sobek. Toutes ces situations révèlent l'importance de l'idéologie lactée égyptienne, qui s'inscrit dans un discours sur le maintien de la vie.

Nous allons nous pencher à présent sur un allaitement insolite, où l'allaité est le souverain et la nourrice une entité divine, souvent thériomorphe.

\section{LE ROI ALLAITÉ PAR UNE ENTITÉ THÉRIOMORPHE}

Différentes déesses bovines participent du discours portant sur la fertilité, la fécondité et la maternité (Hathor, MehetOuret, Ihet, Hésat, Sekhat-Hor, Nout, Akhet, etc.). Cette vache divine, dans ses différents avatars, se montre à la fois créatrice et maternelle. Nourrice, elle remplit alors une fonction logiquement dévolue à la mère. Les dieux qui entretiennent avec elle un rapport de dépendance sont tous impliqués dans le processus de renaissance, que ce soit Osiris ou le jeune dieu solaire. Par ailleurs, la déesse allaitante entretient un rapport particulier avec le roi. "L'allaitement du roi " est en effet un motif symbolique intégré dans les rituels du couronnement (Jean \& Loyrette 2010: 210, 211) consacrant l'accès à la vie royale (Leclant 1951). Dès la Ve dynastie, l'allaitement du roi par une divinité anthropomorphe ou thériomorphe est attesté dans l'iconographie (Ćwiek 2003: 176-183), ainsi sur un fragment du temple de Niouserrê, qui montre le roi allaité par une déesse à tête de lionne (Fig.1 ; 


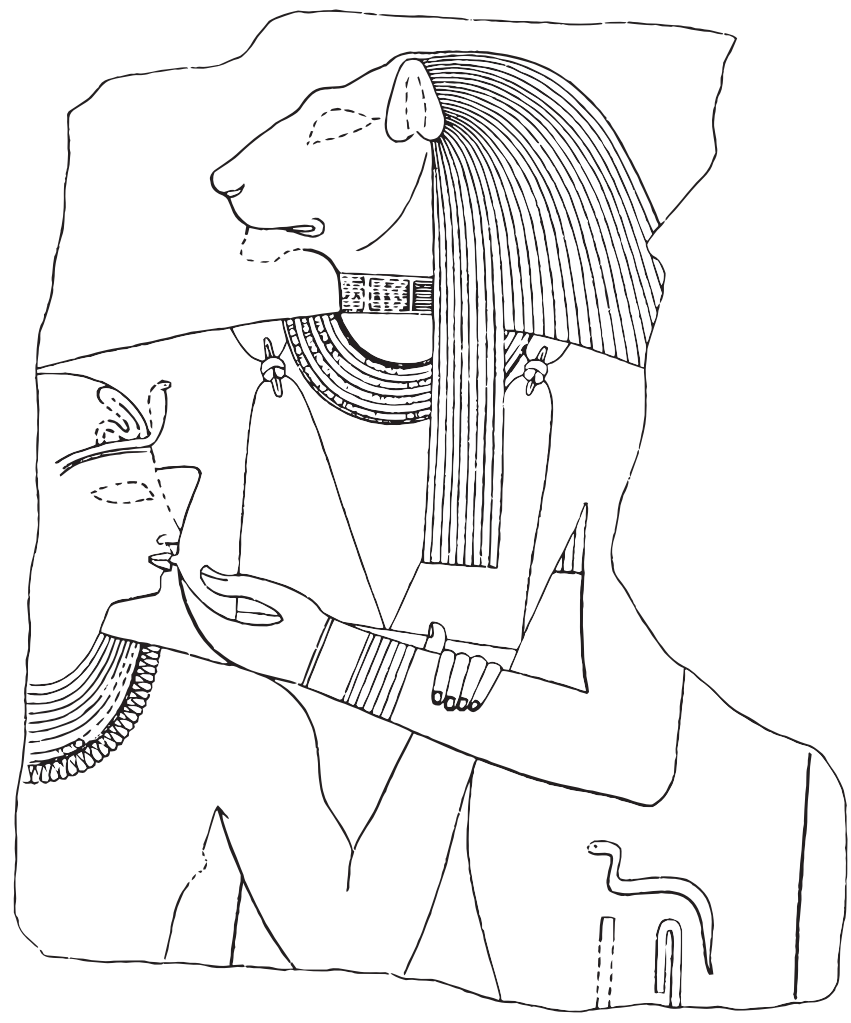

FIG. 1. - La déesse Sekhmet allaitant le pharaon Niouserrê (d'après Borchardt 1907).

Borchardt 1907: 39-41; relief Berlin ÄM no 17911). Dès la XIe dynastie, apparaît un motif iconographique appelé à un grand succès: le roi buvant du lait directement au pis d'une vache, laquelle manifeste sous forme bovine le grand principe féminin (hathorique) du cosmothéisme pharaonique. Le motif du roi allaité par une vache divine se développe notamment à Thèbes, dans le cadre des cultes hathoriques associés à la royauté, qui se consacrent dans un secteur particulier de la nécropole, à Deir el-Bahari (Bietak 2012: 137, 138). Ainsi, le roi Montouhotep II (XIe dynastie) est représenté en train de téter au pis (relief Hanovre Kestner Museum, no1935.200.82, Drenkhahn 1989: 63). Un exemple monumental frappant est offert par une statue du roi Amenhotep II (conservée au Musée du Caire). Cette statue fut découverte par Edouard Naville dans le sanctuaire rupestre d'Hathor de Deir el-Bahari : le pharaon boit à la mamelle de la déesse vache (Fig. 2). Toujours à Deir el-Bahari, les attestations les plus remarquables de cette iconographie royale, sur lesquelles nous allons brièvement nous arrêter, proviennent du temple d'Hathor (règne d'Hatchepsout, XVIII e dynastie) (Beaux 2012). Outre l'iconographie proprement dite, des textes accompagnent ces scènes et précisent la nature de cet allaitement. On lit ainsi dans le discours de la déesse «Hathor Mère-divine (mout-netjer)»:

"Ô ma fille bien aimée Maât-ka-Rê, je t’ai donné toute vie et toute force auprès de moi, en te faisant venir à l'existence (sekheper) par mon lait: car c'est en toute vie et force qu'il entre en toi."
Ce texte informe précisément sur la nature de ce don de vie: le lait fait "venir à l'existence", c'est-à-dire, pour rester proche du sens littéral égyptien, qu'il "transforme» (sekheper). Le lait participe à la construction de la vie: le fluide divin agrège le corps royal. Le thème iconographique du souverain "allaité" connaît un grand succès, et de nombreuses entités divines sont impliquées. Mais l'allaitement n’implique pas seulement les déesses bovines. Par exemple, Ouret-Hékaou, sous forme de cobra anthropomorphisé, peut allaiter le souverain (bijou provenant de la tombe de Toutankhamon); ou encore, Isis, sous forme d'arbre, tend un sein dont se saisit Thoutmosis III, dans une scène fameuse de sa tombe de la Vallée des Rois (KV 34 : la scène est légendée "il tète sa mère Isis"); ici, l'image joue sur le vaste registre symbolique végétal en vertu duquel les fruits deviennent des seins, bons à être tétés (Erroux-Morfin 2009; Jean \& Loyrette 2010: 79-98). Ce qui est donné à voir par cette riche iconographie procède d'un discours symbolique bien défini. Ces images expriment essentiellement une idée sur la transmission, par le vecteur du lait, d'une force vitale divine nécessaire à la conservation de la vie. Il s'agit à présent de questionner la physiologie égyptienne pour comprendre quelle part le lait divin joue dans ce processus.

\section{LE LAIT ET LES CHAIRS}

Les textes associés à l'allaitement royal indiquent que le lait est impliqué symboliquement dans le processus de l'existence, car non seulement il nourrit, mais surtout il «transforme» (sekheper) le corps. Nous aimerions rappeler à présent plusieurs éléments découlant des récits mythologiques, mettant en scène ce processus. La recomposition des chairs est notamment associée à un objet nommé imiout, terme qui signifie littéralement «celui qui est dans les bandelettes" (Fig. 3). Il s'agit d'une peau animale servant d'outre, connue dans les représentations dès les premières dynasties (Köhler 1975; Logan 1990). Du sang en dégouline et coule dans un récipient. On a reconnu que cet objet représente symboliquement le corps du défunt enveloppé dans une enveloppe matricielle (placentaire) (Spieser 2006). Un mythe associé à cet objet est connu grâce au papyrus Jumilhac (IIIe siècle av. J.-C.; Vandier 1961), un compendium des mythes et rites du XVIIIe nome de Haute Égypte. Une historiola y concerne l'objet imiout. Il s'agit de « connaître le secret de la mehet-imiout» («l'outre remplie»). Le mythe évoque le destin tragique du dieu Nemty. De près ou de loin, cet épisode est lié au récit consigné dans un texte plus ancien, le «conte d'Horus et de Seth", préservé par le papyrus Chester Beatty I (XIXe dynastie). Dans ce mythe antérieur, Nemty est un batelier-passeur qui est condamné à être amputé des orteils pour avoir conduit Isis dans l'île du jugement, où se tient le tribunal qui oppose Horus et Seth. Ayant été payé en or par Isis, Nemty est en outre voué à détester cette matière précieuse, conçue comme étant la chair des dieux. Dans le papyrus Jumilhac, les données sont sensiblement différentes. Nemty est coupable cette fois d'un crime majeur, la décapitation d'une déesse vache: 


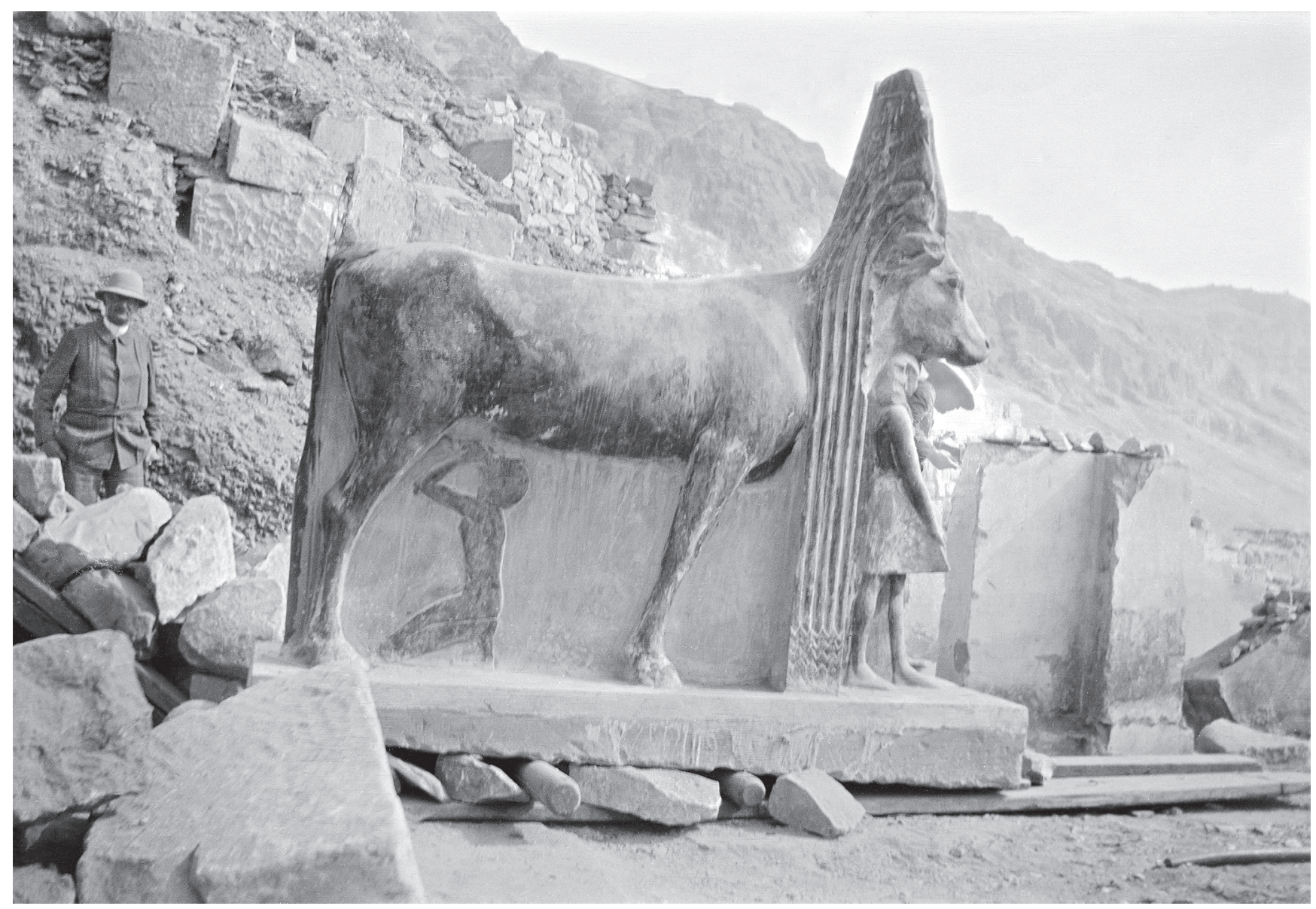

FIG. 2. - La déesse Hathor allaitant le pharaon Amenhotep II, sanctuaire d'Hathor à Deir el-Bahari; photo prise par Marguerite Naville au moment de la découverte de la statue (17.02.1906) @) Musée d'art et d'histoire, Ville de Genève, inv. no A 2006-0030-053-050.

"Quelqu'un avait commis ce crime dans la province de Dounaouy. [Cela] survint dans le Domaine d'Hathor, maîtresse de Mefkat. Rê et la compagnie divine, après l'avoir appris, furent grandement en colère et outragés. Alors Rê dit:

"En ce qui concerne ses chairs (iouf) et sa peau (inem): sa mère les a fait advenir (sekheper) avec son lait. En ce qui concerne ses os (qesou), ils existent par le sperme de son père. Aussi, que l'on éloigne de lui sa peau et ses chairs, et que ses os restent en sa possession".

On fit pareillement dans la province de Djouf. C'est pourquoi l'or est l'aversion dans la province de Djouf, car l'or ce sont ses chairs et l'argent ce sont ses os. On érigea une statue de Nemty en argent à cause de cela (...)».

Le mythe rappelle les associations symboliques divines or/ chair et argent/os, c'est-à-dire une répartition des deux matières précieuses minérales principales entre éléments mous et éléments solides du corps divin. La peau de l'écorché et ses os constituent dès lors l'outre imiout, réceptacle matriciel pour les corps blessés. Ensuite, il faut lire la guérison, par le lait, du corps divin meurtri (Meeks 2006: 181):

"Alors il (Rê) partit et se dirigea vers la province de Dounaouy, avec les dieux à sa suite, Thot étant à leur tête, sa peau (= celle de Nemty) étant avec lui.
Le cour de Hésat fut heureux à cause d'elle. Elle fit de nouveau jaillir son lait pour lui, afin de renouveler sa naissance, et elle fit monter le lait au bout de ses seins, et elle les dirigea vers sa peau, en cet endroit, en y faisant couler le lait. Elle fit, en cet endroit, un onguent dans son godet, et grâce à lui, sa peau et ses chairs furent guéries.

Il y eut une peau à l'intérieur de toute étable, et on y fait couler du lait jusqu'à ce jour (...)».

Le lait prodigué par la déesse vache Hésat, transformé en onguent après barattage, soigne le dieu meurtri et "renouvelle sa naissance»: il guérit, il ressuscite les chairs. Le mythe rappelle la fonction physiologique du breuvage; en outre, il fonde et promet l'abondance laitière, dans " toute étable ", où le lait coulera désormais à flots.

\section{LA CHAIR ET LES OS, LE LAIT ET LE SPERME}

Le discours égyptien sur la création du corps humain apporte quelques éléments intéressants, notamment en ce qui concerne la constitution des chairs et des fluides corporels. Une conception attestée en tous cas depuis l'époque perse (la plus ancienne attestation provient du temple d'Hibis, sous le règne de Darius II, vers 500-485 av. J.-C.) voulait que le 


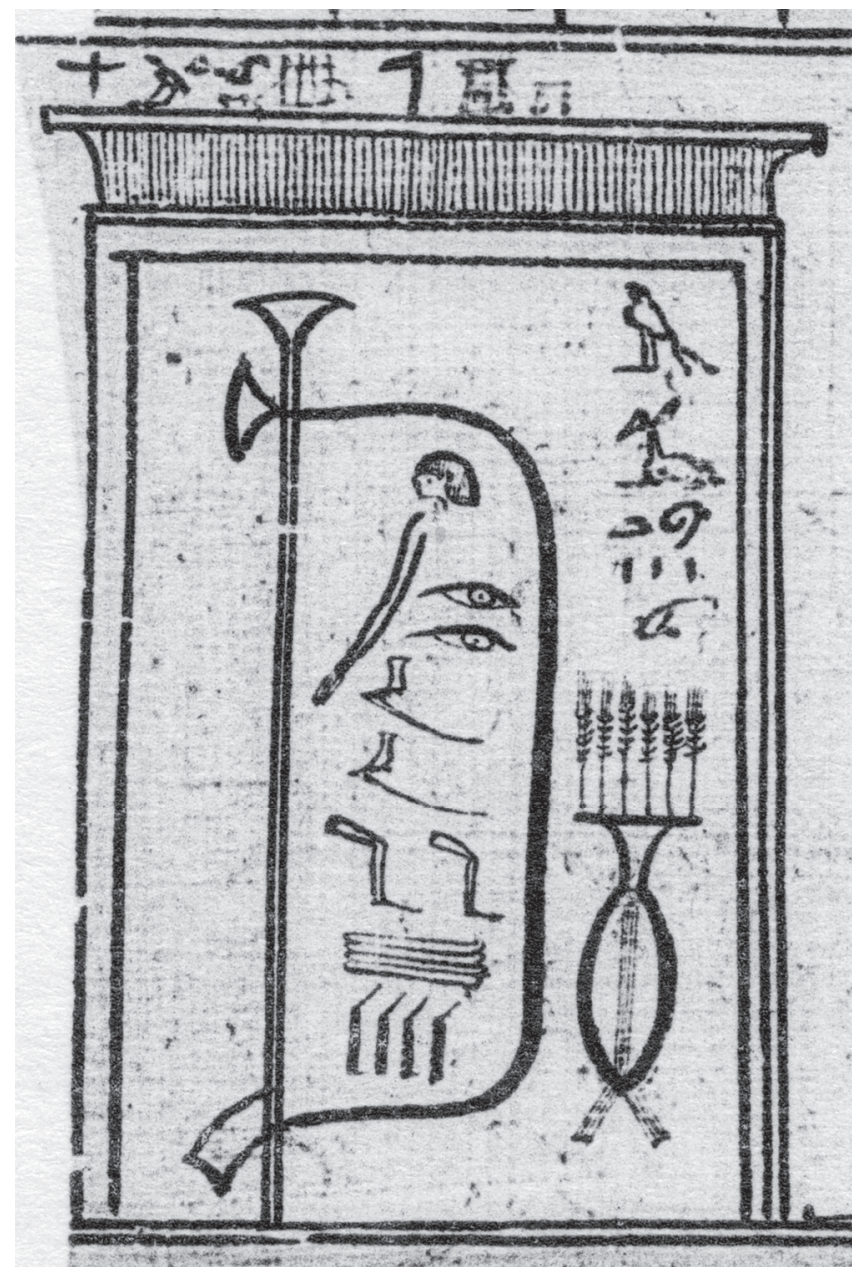

FIG. 3. L'outre-imiout, réceptacle du corps divin blessé; d'après Vandier 1961, p. Louvre E 17110, page V.

dieu créateur, architecte du corps des humains, ait conçu les os comme réservoir du sperme. Serge Sauneron (1960), qui avait mis en lumière cette notion, avait supposé qu'une conception grecque analogue, connue depuis le présocratique Hippon de Samos (Ve siècle av. J.-C.) (idée réfutée plus tard par Aristote, cf. Bonnard 2004: 175-177), posant elle aussi que le sperme venait de la moelle, avait pu influer sur l'idée égyptienne. Néanmoins, on peut penser qu'il s'agit plutôt de l'inverse, c'est-à-dire que l'idée exprimée par les textes égyptiens ait été secondairement relayée par les Grecs. Jean Yoyotte (1962), poussant l'investigation, a montré qu'il s'agissait de comprendre que la physiologie égyptienne, s'appuyant sur des données exprimées notamment dans les textes sacerdotaux, avait conçu un lien organique entre le phallus et la colonne vertébrale, en pensant un organe menehep formé par le dos et la colonne vertébrale, une épine dorsale qui se révèle, à la lecture des textes, être l'appareil génital d'Osiris (Bardinet 1995: 139-153). Au niveau anthropologique, Jean Yoyotte en déduisait que ces idées révélaient "une interprétation rationnelle tirée de l'observation immédiate des faits bruts", "la capsule fermée des os " comme "endroit idéal» du stockage de la semence (Yoyotte 1962: 151), laquelle observation participait de l'universalité des discours sur la consubstantialité entre le sperme et la moelle. Cette vision égyptienne a été remarquée par Plutarque.

Pour ce dernier, qui prétend s'appuyer sur un mythe égyptien selon lequel Horus aurait été puni de mutilation après avoir tué sa mère Isis, ce dieu aurait eu en conséquence le corps curieusement mutilé: son sang et sa moelle (reçus par transmission paternelle) lui auraient été laissés, alors que sa graisse et ses chairs (transmission maternelle) lui auraient été arrachées (Hopfner 1922: 266 ; Ziegler \& Pohlenz 1953: 42 ; Yoyotte 1962: 144).

On ne peut relier directement le récit de Plutarque à un seul mythe précis, néanmoins, à l'exception de la transmission du sang par voie paternelle, les éléments qu'il assemble sont bien conformes à plusieurs récits égyptiens, notamment à des données analogues à celles du papyrus Chester Beatty I (le "Conte d'Horus et de Seth»), et ouvre sur la physiologie des chairs, des os et du sang. Les anthropologues n'ont pas manqué de s'intéresser à ces données égyptiennes, notamment Françoise Héritier, qui se base sur les travaux de Serge Sauneron et de Jean Yoyotte (Héritier 1996: 146149), de même que Salvatore D’Onofrio (2014: 148). Pour Françoise Héritier, la question des diffusions de savoir entre l'Égypte et le monde méditerranéen sur la question précise du partage particulier entre masculin et féminin dans l'hérédité passe au second plan; l'origine de ce type de croyance, portant sur le stockage de la semence dans le corps à la convergence entre colonne vertébrale et pénis, constatée dans des points divers du globe, serait « somme toute (...) une interprétation rationnelle tirée de l'observation des faits bruts» (Héritier 1996: 151) constituant un système universel de pensée. Dans une belle étude centrée sur le monde italien, D'Onofrio reconnaît en Sicile « une configuration de type égyptien de la théorie de la semence dans les os" (D’Onofrio 2014: 161). Cette physiologie égyptienne de l'hérédité offre à l'anthropologue l'image d'une société associant à parts égales les contributions masculine et féminine à la création du corps humain. On peut tenter de dresser un tableau schématique pour illustrer ces frontières sexuées du corps pensées par l'idéologie égyptienne (Tableau 2).

Dans les discours cosmogoniques, le créateur, le dieu unique (issu du Noun primordial), est volontiers conçu comme androgyne, "père et mère " simultanément de la création. À l'instar du moment cosmogonique, qui inaugure un processus de différenciation permanent, la genèse du corps humain, qui repose fondamentalement sur l'action directe du dieu créateur à l'intérieur du corps de la femme (comme l'expose par exemple un fameux texte du temple d'Esna, voir, en dernier lieu, Mathieu 2012), est un processus par étapes. Tout comme le sperme consiste en une dilution de l'os paternel, le lait est conçu comme une dilution de la chair maternelle (Bardinet 1995: 145). Durant l'allaitement, le don du lait prolonge, après la naissance, l'agrégation des éléments mous. Quant à l'autre fluide qui joue un rôle central, le sang, lui aussi reçu de la mère, il agrège les éléments solides du corps. 


\section{CONCLUSIONS}

Le thème de l'allaitement nous a amenés à évoquer la conception égyptienne de l'hérédité dans sa transmission genrée, qui n'établit pas de manière indiscutablement marquée une suprématie masculine sur l'ensemble de l'édifice corporel. Loin de là, il semble d'ailleurs que la part maternelle dans la constitution et la formation du corps est non seulement importante, mais encore appelée à un rôle fondamental dans la survie post mortem. On peut illustrer ce fait en rappelant une formule funéraire du Livre des Morts (chapitre 30b; Malaise 1978). Cette formule, très courante, est reprise dès le Nouvel Empire sur les «scarabées de cour» (Malaise 1978).

Formule pour empêcher que l'on enlève le cœur-jb de Nebseni, juste-de-voix (...):

"Ô cour-jb [venant] de ma mère, ô cour-jb [venant] de ma mère, ô cour-hâty de mon existence, ne te lève pas contre moi en témoignage, ne t’oppose pas à moi devant le tribunal, ne montre pas d'hostilité contre moi en présence du gardien de la balance! Tu es mon ka, qui est dans mon corps, le Khnoum qui rend prospères mes membres [...]» (papyrus de Nebseni ; Lapp 2004: pl. 12).

Le cœur-jb-le «for intérieur» - et le cœur-hâty - le muscle cardiaque - sont tous deux convoqués pour la survie dans l'audelà; plus précisément, on envisage leur rôle dans le jugement des morts, lors duquel le «cœur» est censé représenter la somme des actions de l'homme, symboliquement pesée devant lui. La finalité de ce passage de la formule du chapitre 30b est de s'assurer que le "cœur» ne va pas se retourner contre le défunt, en révélant quelque faute indicible. Car cet organe cardiaque joue un rôle central dans les conceptions égyptiennes; l'intérieur-jb est en somme l'organe intellectuel et émotionnel, tout à la fois conscience et pensée; il est également la mémoire de l'être. Ce cour est bien "le centre de l'homme» (Assmann 1993). Si la transmission d'un cour/intérieur-jb directement du dieu père au roi est attestée (Bardinet 1995: 146, 147), néanmoins, le chapitre $30 \mathrm{~b}$ insiste sur le fait que l'intérieur-jb et le cœur-hâty, éléments "mous", sont bien des dons de la mère, transmis puis fondamentalement formés lors de l'allaitement. Le « cœur venant de la mère", comme aime à le rappeler la formule du Livre des Morts, est bien le témoin ultime de l'individu. Le lait et le sang, dons de la mère, accompagnent ainsi l'homme du berceau à la tombe.

\section{Remerciements}

Je remercie cordialement Jean-Luc Chappaz, conservateur en chef au Musée d'Art et d'Histoire de Genève, pour l'autorisation de publication de la photographie tirée des archives Naville. Mes remerciements s'adressent également à Cathie Spieser et Pierre Tallet pour leur relecture attentive.

\section{RÉFÉRENCES}

Assmann J. 1993. — Zur Geschichte des Herzen im Alten Ägypten, in Assmann J. \& Sundermeier T. (éds), Die Erfindung des Inneren Menschen: Studien zur religiösen Anthropologie. Studien zum Verstehen fremder Religionen 6: 81-113.

BARDINET T. 1995. - Les papyrus médicaux de l'Égypte pharaonique. Fayard, Paris, 583 p.
TABLEAU 2. - Schématisation des frontières sexuées du corps pensées par l'idéologie égyptienne.

\begin{tabular}{|c|c|}
\hline Transmission maternelle & Transmission paternelle \\
\hline \multicolumn{2}{|c|}{$\begin{array}{l}\text { Par le biais } \\
\text { du sp }\end{array}$} \\
\hline Relation sym & $\begin{array}{l}\text { bolique } \\
\text { Argent }\end{array}$ \\
\hline $\begin{array}{l}\text { Chairs - Éléments mous } \\
\text { Chair (iouf) } \\
\text { Peau (inem) } \\
\text { Organes internes du tronc, } \\
\text { «ventre» (chet) } \\
\text { Veines, artères (conduits-met) } \\
\text { Sang }\end{array}$ & $\begin{array}{l}\text { Os - Éléments solides } \\
\text { Os (qésou) } \\
\text { Dents (ibéhou) } \\
\text { Ongles (anout) } \\
\text { Cheveux (shénou) } \\
\text { Poils et barbe (sérou) }\end{array}$ \\
\hline Cœur & \\
\hline
\end{tabular}

BEAUX N. 2012. - La chapelle d'Hator: temple d'Hatchepsout à Deir el-Bahari. I: Vestibule et sanctuaires. MIFAO 129, 3 vol.: 260 + $108+188$ p. + 1 CD-Rom.

BIETAK M. 2012. — La belle fête de la Vallée: l'Asasif revisité, in Zivie-Coche C. \& Guermeur I. (éds), "Parcourir l'éternité", Hommages à Jean Yoyotte. Bibliothèque de l'École des Hautes Etudes. Sciences religieuses 156: 135-164.

Bonnard J. B. 2004. - Le complexe de Zeus. Représentations de la paternité en Grèce ancienne. Sorbonne, Paris, 254 p. (Coll. Histoire ancienne et médiévale; 76).

BorCHARDT L. 1907. — Das Grabdenkmal des Königs Ne-User-Re . Leipzig, iii + 184 p. +27 pl.

BoysSON B. DE \& SPURR S. 2007. — Égypte: trois mille ans d'art décoratif. Musée Myers. Collection du Collège d'Eton. Somogy; Musée des Arts décoratifs, Bordeaux, 80 p.

ĆWIEK A. 2003. - Relief Decoration in the Royal Funerary Complexes of the Old Kingdom. Ph.D. Thesis, Institute of Archaeology, Warsaw University.

D'OnOFRIO S. 2014. — Les fluides d'Aristote: lait, sang et sperme dans l'Italie du Sud. Les Belles Lettres, Paris, 192 p. (Coll. Vérité des mythes; 42).

DRENKHAHN R. 1989. - Ägyptische Reliefs im Kestner-Museum Hannover. Kestner Museum, Hannover, 154 p.

El SAYed R. 1982. - La déesse Neith de Saïs. II: Documentation. IFAO, Le Caire, 715 p. +15 pl. (Coll. Bibliothèque d'Études; 86).

ErrouX-Morfin M. 2009. - Du lait-blanc à l'orgeat de souchet, in RÉGEN I. \& SERVAJEAN F. (éds), Verba Manent. Recueil d'études dédiées à Dimitri Meeks. Cahiers Égypte Nilotique et Méditerranéenne 2: 125-135.

Grandet P. 1998. - Contes de l'Égypte ancienne. Hachette, Paris, $196 \mathrm{p}$.

Griffith F. L. \& Newberry P. 1893. - Beni Hasan. Part II. Archaeological Survey of Egypt, Londres, 87 p. +38 pl.

Griffith F. L. \& Newberry P. 1894. - El Bersheh. Part II. Archaeological Survey of Egypt, Londres, 70 p. +23 pl.

Guglielmi W. 1982. — s.v. Milch(wirtschaft), in HelcK W. \& Oтто E. (éds), Lexikon der Ägyptologie IV: Meggido-Pyramiden. Harrassowitz, Wiesbaden: 125-127.

Harpur Y. \& SCREMIN P. 2006. - The Chapel of Kagemni. Scene Details. Oxford Expedition to Egypt, Oxford, 544 p. (Coll. Egypt in Miniature; 1).

HÉRITIER F. 1996. - Masculin / Féminin : la pensée de la différence. Odile Jacob, Paris, 332 p.

Hopfner T. 1922. - Fontes Historiae Religions Aegyptiacae. Marcus \& Weber, Bonn, 932 p.

JEAN R. A. \& LoyretTe A.-M. 2010. — La mère, l'enfant et le lait en Égypte ancienne. L'Harmattan, Paris, 516 p. (Coll. Kubaba Série Antiquité, Université de Paris I Panthéon Sorbonne). 
Kanawati N. \& Woods A. 2010. — Beni Hassan. Art and Daily Life in an Egyptian Province. Supreme Council of Antiquities, Le Caire, 200 p.

KÖHLER U. 1975. - Das Imiut: Untersuchungen zur Darstellung und Bedeutung eines mit Anubis verbundenen religiösen Symbols. Harassowitz, Wiesbaden, xv + 529 p. (Coll. Göttinger Orientforschungen; IV: Ägypten).

LAPP G. 2004. - Catalogue of the Books of the Dead in the British Museum. Vol. III: The Papyrus of Nebseni. Trustees of the British Museum, London, 100 p. $+100 \mathrm{pl}$.

LECLANT J. 1951. - Le rôle du lait et l'allaitement d'après les Textes des Pyramides. Journal of Near Eastern Studies 10 (2): 123-127. http://www.jstor.org/stable/542260

LefebVRe G. 1960. — Lait de vache et autres laits en Égypte. Revue d'égyptologie 12: 59-65.

LOGAN T. 1990. - The origins of the imy-wt fetish. Journal of the American Research Center in Egypt 27: 61-69.

MALAISE M. 1978. - Les scarabées de cour dans l'Égypte ancienne, avec un appendice sur les scarabées de coeur des Musées royaux d'art et d'histoire de Bruxelles. Fondation égyptologique Reine Élisabeth, Bruxelles, 94 p.

Mathieu B. 2012. - Et tout cela exactement selon sa volonté: la conception du corps humain (Esna no250, 6-12), in GASSE A., SERVAJEAn F. \& Thiers C. (éds), Et in Aggypto et ad Egyptum: recueil d'études dédiées à Jean-Claude Grenier. Cahiers Égypte Nilotique et Méditerranéenne 5 (3): 499-516.

MEEKS D. 2006. - Mythes et légendes du Delta d'après le papyrus Brooklyn 47.218.84. IFAO, Le Caire, 498 p.

Millet J. 1987. - Un allaitement insolite, in HaINARD J. \& KAEHR R. (éds), Des animaux et des Hommes. Musée d'ethnographie, Neuchâtel: 87-118.

Quaegebeur J. 1977. - Anubis, fils d'Osiris, le vacher. Studia Egyptiaca 3: 119-130.
Rigault P. 2013. — Scènes d'allaitement: de la scène de genre à la composition symbolique, in ANDREU-LANOË G. (éd.), L'art $d u$ contour. Le dessin dans l'Égypte ancienne. Louvre; Somogy Editions d'art, Paris: 96-101.

SAUNERON S. 1960. - Le germe dans les os. Bulletin de l'Institut Français d'Archéologie Orientale 60: 19-27.

SETHE K. 1908-1922. - Die altaegyptischen Pyramidentexte. Hinrich, Leipzig, 4 vols: 510 p. +543 p. +179 p. +130 p.

SHedid A. G. 1994. - Die Felsengräber von Beni Hassan in Mittelägyptien. Philipp von Zabern, Mainz, iv + 96 p.

SPIESER C. 2006. - Vases et peaux animales matriciels dans la pensée religieuse égyptienne. Bibliotheca Orientalis 63 (3): 219-234. https://doi.org/10.2143/BIOR.63.3.2017972

SPIESER C. 2014. - La nature ambivalente du sang, du lait, des figues et du miel dans les croyances funéraires égyptiennes, in TALLET G. \& ZIVIE-COCHE C. (éds), Le myrte \& la rose: mélanges offerts à Françoise Dunand par ses élèves, collègues et amis. Cahiers Egypte Nilotique et Méditerranéenne 9 (2): 281-287.

Tran Tam Tinh V. 1973. - Isis lactans. Corpus des monuments grécoromains d'Isis allaitant Harpocrate. Brill, Leiden, xii +225 p. + $78 \mathrm{pl}$. (Coll. Études préliminaires aux religions orientales dans l'Empire romain; 37).

VANDIER J. 1961. - Le papyrus Jumilhac. CNRS Éditions, Paris, 350 p. VOlOKHINE Y. 2014. - Le porc en Égypte ancienne. Mythes et histoire à l'origine des interdits alimentaires. Presses Universitaires de Liège, Liège, $306 \mathrm{p}$.

YOYOTTE J. 1962. — Les os et la semence masculine. À propos d'une théorie physiologique égyptienne. Bulletin de l'Institut Français d'Archéologie Orientale 61: 139-146.

Ziegler K. \& Pohlenz M. 1953. - Plutarchi Moralia. Vol. VI, fasc. 3. Teubner, Leipzig, $50 \mathrm{p}$. 